\title{
Prior probability variation in an information purchase task ${ }^{1}$
}

\section{RICHARD CHAPMAN ${ }^{2}$ and JOSEPH HALPERN, ${ }^{3}$ University of Denver, Denver, Colo. 80210}

The prior probabilities were varied for two hypotheses in an informationpurchasing task in which Ss were required to construct the prior probabilitygenerating device personally. Biases in priors did not affect the number of pieces of data purchased. Comparison of subjective opinion revisions with the Bayesian normative model showed that $S$ s were less conservative with data having high diagnostic impact than they were with data having low diagnostic impact. No performance differences were evident for infirming and confirming forms of data.

A number of recent experiments have shown that $\mathrm{Ss}$, in decision-making situations, consistently demonstrate conservative behavior both in the formation of expectancies and in the processing of information. In the case of expectancies, when Ss make a decision in favor of one of several relevant states of the world, they will frequently assume subjective biases regarding the relative likelihood of occurrence of these states that are less than those objectively present in the data-generating system (Pitz, in press; Chapman, 1969). These biases or expectancies are referred to as prior probabilities.

A second form of conservatism is reliably observed when Ss revise their prior probability biases with respect to information pertaining to the relevant states of the world. Specifically, when Bayes's Theorem is used as a normative model for the revision of probabilistic opinion as a function of information, it is possible to evaluate the optimality of the decision-making behavior demonstrated by Ss. Bayes's Theorem may be expressed in odds form as $\Omega_{1}=L \Omega_{0}$, where $\Omega_{1}$ is the posterior odds in favor of $\mathrm{H}_{1}$ over $\mathrm{H}_{\mathrm{o}}$ on the basis of the datum $D_{1}, \Omega_{0}$ is the prior odds in favor of $\mathrm{H}_{1}$ over $\mathrm{H}_{0}$, and $\mathrm{L}$ is the likelihood ratio (i.e., the ratio of the probability of the datum given $\mathrm{H}_{1}$ to the probability of the datum given $\mathrm{H}_{\mathrm{o}}$ ). The likelihood ratio corresponds to the diagnostic impact of the datum.

The literature indicates that $\mathrm{Ss}$ tend to lag behind the normative model in the amount of opinion revision occasioned by a given datum. In some cases, Ss have been observed to perform in a more optimal manner when the datum processed is infirming information, e.g., data which provides evidence contrary to the prior probability bias, than when the datum is confirming or consonant with the prior probability bias (Chapman, 1969).

The present experiment was designed to further investigate conservative behavior in a decision-making task in which Ss were required to purchase the data necessary for each decision. It was hypothesized that, when Ss were given the opportunity to ascertain prior probability bjases by personally building them into the data-generating system, they would purchase less data for decisions made in favor of the most frequently occurring state of the world, $\mathrm{H}_{1}$.

\section{SUBJECTS}

Forty female introductory psychology students at the University of Denver were assigned randomly to four groups of $10 \mathrm{Ss}$ each.

\section{MATERIALS}

Two decks of 100 computer cards each were employed. Fifty letters, chosen from a population of letters, including " $A$ " through " $F$," were interpreted on the top of the cards. These letters were sampled randomly from one of the two distributions of letters given in Table 1. The printed face of each card was color-coded to correspond to one of the two distributions of letters. Thus, the cards in the deck sampled from the red distribution were marked with a red watercolor bar on the printed face, and the cards in the deck sampled from the black distribution were similarly marked with a black bar. A chart identical to Table 1 was placed in full view of the $S$ at the beginning of the experimental session.

Two probability bars were used for subjective probability estimates. Both bars were 14-in. wooden dowels supported by two $1 \times 3 \times 4$ in. blocks. Each dowel was marked off in 10ths from .50 to .99 . A

Table 1

Distributions of Data in the Red and Black States of the World

\begin{tabular}{lcc}
\hline & Red & Black \\
Letter & $\begin{array}{c}\text { Probability } \\
\text { of Sampling }\end{array}$ & $\begin{array}{c}\text { Probability } \\
\text { of Sampling }\end{array}$ \\
\hline $\mathrm{A}$ & .03 & .07 \\
$\mathrm{~B}$ & .30 & .10 \\
$\mathrm{C}$ & .30 & .20 \\
$\mathrm{D}$ & .20 & .30 \\
$\mathrm{E}$ & .10 & .30 \\
$\mathrm{~F}$ & .07 & .03 \\
\hline
\end{tabular}

$3 \times 5$ in. marker card was used to indicate probability values on the bars. One bar was designated "Black Probability," and the other was labeled "Red Probability."

Poker chips were used as monetary symbols, with blue chips standing for $5 \downarrow$ and yellow chips representing $1 / 24$. Chips accumulated by Ss were turned in for cash at the end of the experiment.

\section{PROCEDURE}

The $S$ was told that the task involved a decision between two states of the world, red and black. The $S$ was required to predict the color of the top card in a shuffled deck of 60 red and black cards hidden face down behind a screen. A correct prediction was rewarded $15 \phi$, while an incorrect response resulted in a $15 \$$ loss. In order to reduce the uncertainty involved in each decision, $S$ was allowed to purchase data at a cost of $1 / 24$ per datum. Following the first purchase, the first letter interpreted on the top of the computer card in question was read. The $S$ then consulted a chart similar to Table 1 in order to gain certainty about one of the two states of the world. Each $S$ was permitted to purchase as many data as desired before arriving at a final decision. Subjective probability estimates were obtained after each purchase. The computer card was presented to $S$ for examination after each final decision. This final decision marked the end of a trial, and 50 trials were given to each $S$ in two sets of 25 trials each.

In order to vary prior probabilities, the experimental session was initiated by showing S two decks of 100 cards each, one red and one black. The $S$ was then instructed to build a new deck of 60 mixed cards from the two separate decks according to E's specification. At the end of 25 trials, $S$ was required to put all of the cards back into the respective separate color decks. A new deck of 60 mixed cards was then recreated according to E's specification.

Group 1 experienced unbiased prior probabilities, i.e., the probability of a red card, $P\left(\mathrm{H}_{1}\right)$, was equal to the probability of a black card, $\mathrm{P}\left(\mathrm{H}_{2}\right)$, or .50 , on both the first 25 trials and the second 25 trials. Group 2 experienced biased priors $\left[\mathrm{P}\left(\mathrm{H}_{1}\right)=.70, \mathrm{P}\left(\mathrm{H}_{2}\right)=.30\right]$ on both sets of trials. Group 3 initially experienced unbiased priors so that $\mathrm{P}\left(\mathrm{H}_{1}\right)=.50$ for the first 25 trials but was shifted to biased priors $\left[P\left(\mathrm{H}_{1}\right)=.70\right]$ for the second set of 25 trials. The last group, Group 4, experienced a shift from biased priors $\left[P\left(H_{1}\right)=.70\right]$ to unbiased priors $\left[\mathrm{P}\left(\mathrm{H}_{1}\right)=.50\right]$.

\section{RESULTS AND DISCUSSION}

Table 2 shows the result of prior probability variation on the number of 
Fig. 1. Optimality of performance as a function of diagnostic impact for all Ss.

pieces of data purchased for decisions on which $\mathrm{S}$ favored $\mathrm{H}_{1}$. It was expected that Ss experiencing biased prior probabilities $\left[\mathrm{P}\left(\mathrm{H}_{1}\right)=.70\right]$ would purchase less data to arrive at an $\mathrm{H}_{1}$ decision than $\mathrm{Ss}$ experiencing unbiased priors $\left[\mathrm{P}\left(\mathrm{H}_{1}\right)=.50\right]$. In order to evaluate the preshift data, Group 1 was combined with Group 3 and Group 2 was combined with Group 4 to provide a between-group comparison of $\mathrm{P}\left(\mathrm{H}_{1}\right)=.50$ and $\mathrm{P}\left(\mathrm{H}_{1}\right)=.70$ Ss. A $t$ test showed that the means were not significantly different.

The postshift data were analyzed via an analysis of variance in which two between-Ss variables were defined: shift in bias vs no shift in bias and $P\left(H_{1}\right)=.50$ vs $P\left(H_{1}\right)=.70$. All differences were insignificant, and thus the procedure used here, which was designed to provide Ss with an awareness of bias without extended experience over trials, was unsuccessful. That is, the hypothesis that Ss would purchase less information on $\mathrm{H}_{1}$ decisions for the biased priors conditions was not supported. This suggests that $S$ s require extended experience with biased priors (Pitz, in press; Chapman, 1969) in order to adopt a subjective bias. The Ss seemed quite unaffected by the experience of personally building prior probability biases into the data-generating system.

The optimality of S's information-processing behavior may be evaluated by creating a ratio of the amount of probabilistic revision shown by $S$ to the amount of revision demonstrated by a hypothesized Bayesian decision maker using the normative model. The optimality ratios for all of the $S$ s across all 50 trials are shown in Fig. 1. Letters A-F, B-E, and C.D are combined since they have equivalent diagnostic impact.

It is of interest to note that performance was nearly Bayesian for the data with the strongest diagnostic impact (Letters C-D)

Table 2

Mean Number of Pieces of Data Purchased for an $\mathrm{H}_{4}$ Decision

\begin{tabular}{lcc}
\hline & $\begin{array}{c}\text { First } \\
\text { 25 Trials }\end{array}$ & $\begin{array}{c}\text { Second } \\
\text { 25 Trials }\end{array}$ \\
\hline Group 1 & 3.35 & 3.45 \\
Group 2 & 3.05 & 3.40 \\
Group 3 & 3.95 & 3.55 \\
Group 4 & 3.45 & 4.40 \\
\hline
\end{tabular}

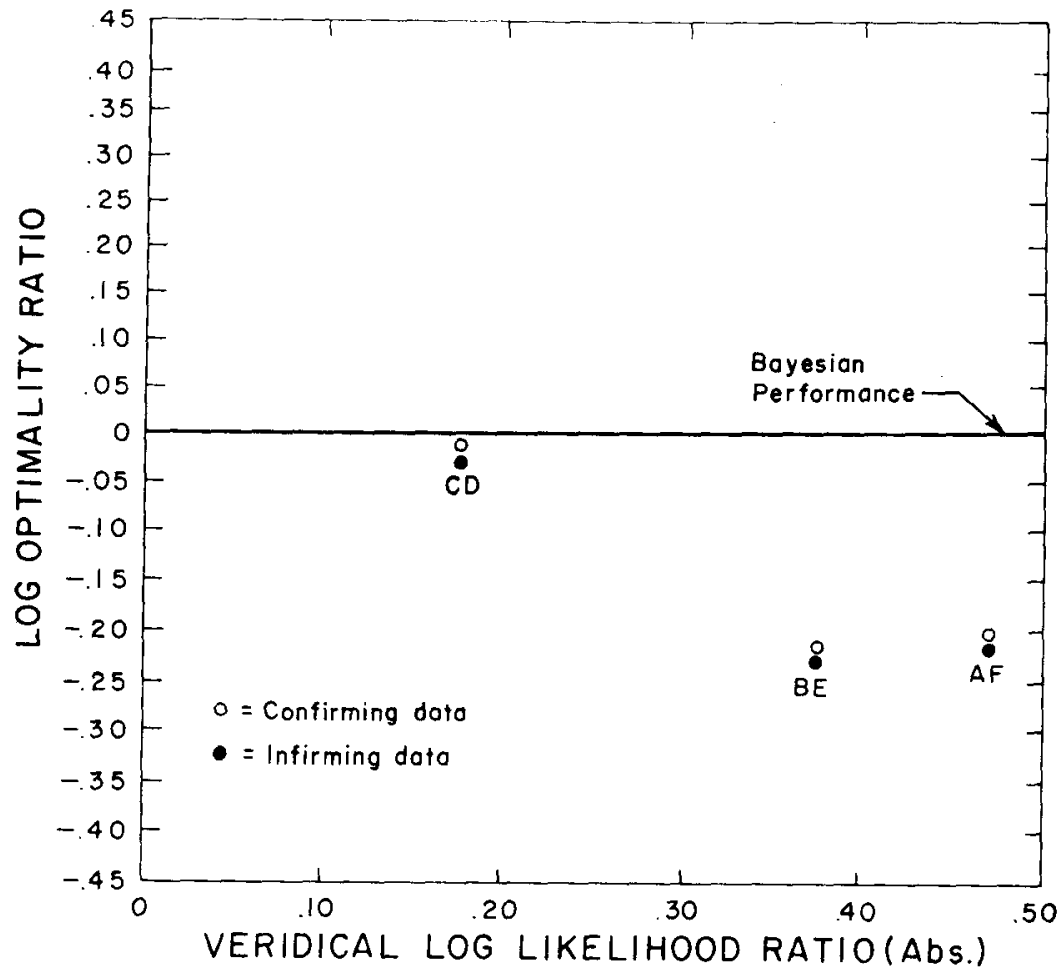

but highly conservative for the other types of data (Letters A-F, B-E). This implies that Ss depended very heavily on $C$ and $D$ data in arriving at a decision and very little on the other kinds of information. It is also important to note that a linear relationship is not evident between conservatism and diagnostic impact. The Ss apparently treated the two weaker forms of data as though they were equivalent. This is surprising in view of recent research with multinomial probability distributions (Schum \& Martin, 1968), where conservatism was found to be linearly related to diagnostic impact of the information processed.

Surprisingly, the data show that Ss performed almost identically for both infirming and confirming types of information. Chapman (1969), using a similar task in which Ss learned biased prior probabilities through probability learning, observed that $S$ s consistently processed infirming data in a more optimal fashion than they did confirming data. Since the present results suggest that $S$ s were not attending to the prior probability biases, it seems that Ss weight infirming data more heavily than they do confirming data only when they are attending to a bias in prior probabilities. It appears that when Ss are aware of a bias, they focus most of their attention on information that is contrary to it. In the absence of a perceived bias, however, Ss do not appear to discriminate between infirming and confirming forms of information.

\section{REFERENCES}

CHAPMAN, C. R. The acquisition and use of biased prior probabilitics in complex information sceking. Unpublished doctoral dissertation, University of Denver, 1969.

PITZ, G. F. The influence of prior probabilities on information seeking and decision making. Organizational Behavior \& Human Performance, in press.

SCHUM, D. A., \& MARTIN, D. W. Human processing of inconclusive evidence from multinomial probability distributions. Organizational Behavior \& Human

$$
\text { NOTES }
$$

1. This research was done during the first author's tenure as a NASA predoctoral fellow at the University of Denver.

2. Now at the Center for the Study of Aging and Human Development, Duke University Medical School.

3. Reprint requests should be sent to Joseph Halpern, Department of Psychology, University of Denver, Denver, Colo. 80210. Performance, 1968, 3, 353-365. 